\title{
Overexpression of astrocyte-elevated gene- 1 is associated with cervical carcinoma progression and angiogenesis
}

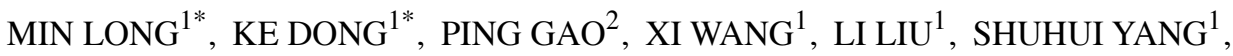 \\ FANG LIN $^{1}$, JUNXIA WEI ${ }^{1}$ and HUIZHONG ZHANG ${ }^{1}$ \\ Departments of ${ }^{1}$ Medical Laboratory, and ${ }^{2}$ Gynecology and Obstetrics, Tangdu Hospital, \\ Fourth Military Medical University, Xi'an, Shaanxi 710038, P.R. China
}

Received April 15, 2013; Accepted May 28, 2013

DOI: $10.3892 / o r .2013 .2598$

\begin{abstract}
Astrocyte-elevated gene-1 (AEG-1) is implicated in the oncogenesis and angiogenesis of various types of human cancers. However, the biological roles of AEG-1 in cervical carcinoma remain to be further elucidated. In the present study, we demonstrated that the expression of AEG-1 was markedly upregulated in the cervical carcinoma cell lines HeLa, CaSki and SiHa, as well as in 8 paired primary cervical carcinoma tissue (CCT) specimens at both the transcriptional and translational levels when compared with normal cervical epithelial cells (NCECs). Furthermore, immunohistochemical (IHC) analysis demonstrated that 180 of 200 (90\%) archived CCT specimens exhibited positive staining for AEG-1, and statistical analysis revealed that the upregulation of AEG-1 was significantly correlated with the clinical staging of the patients $(\mathrm{P}=0.034)$, including $\mathrm{T}(\mathrm{P}=0.019), \mathrm{N}(\mathrm{P}=0.038)$ and $\mathrm{M}$ classification $(\mathrm{P}=0.018)$ as well as tumor differentiation $(\mathrm{P}=0.043)$. Furthermore, loss- and gain-of-function results showed that knockdown of AEG-1 expression by specific shRNA not only inhibited SiHa cell proliferation and invasive ability, but also significantly decreased the expression of the angiogenesis-related genes HIF-1 $\alpha$, Tie2, VEGF and TEMI/ $C D 248$. Moreover, an increased vascular formation ability was observed in human umbilical vein endothelial cells (HUVECs) co-cultured with conditioned medium both from SiHa cells and NCECs transfected with ectopic AEG-1. In conclusion, these results suggest that elevated expression of AEG-1 plays an important role in the aggressiveness and angiogenesis of cervical carcinoma and that AEG-1 represents a novel and valuable predictive factor for the prognostic evaluation of cervical carcinoma patients.
\end{abstract}

Correspondence to: Professor Huizhong Zhang, Department of Medical Laboratory, Tangdu Hospital, Fourth Military Medical University, Xinsi Road, Xi'an, Shaanxi 710038, P.R. China

E-mail: tdzhz@yahoo.com.cn

*Contributed equally

Key words: AEG-1, cervical carcinoma, angiogenesis, tumor marker

\section{Introduction}

Cervical carcinoma is the third most common malignancy affecting women worldwide (1). With the development of surgery and the utilization of radiation and chemotherapy, the death rate has been reduced; however, some tumors are more refractory to treatment, and certain patients are still less sensitive to recently developed treatments (2). Many clinicopathological parameters, including the clinical stage of the disease, tumor volume and metastatic status, have been reported to be associated with prognosis, while they are not directly correlated with tumor aggressiveness. Further research is needed to identify more effective prognostic markers for the improvement of the clinical management of cervical carcinoma patients. This would also support the development of novel individually tailored strategies for use in the treatment of high-risk patients due to molecular risk factors (3-6).

Astrocyte-elevated gene-1 (AEG-1), also known as metadherin (MTDH), was initially identified as a human immunodeficiency virus (HIV)-1-inducible gene in human fetal astrocytes $(7,8)$. AEG-1 has recently received considerable attention in an increasing spectrum of tumor indications due to its multiple roles in regulating cancer progression and metastasis (9). Aberrant elevation of AEG-1 expression frequently occurs in human cancers, including salivary gland, glioma, hepatocellular, esophageal squamous cell and renal cell carcinomas, as well as breast, prostate, melanoma, gastric and non-small cell lung cancers (10-18), which correlates with poor clinical outcomes. Loss- and gain-of-function studies revealed the importance of AEG-1 in regulating multiple signal transduction pathways and various pathologically relevant processes (19-26). AEG-1 has been reported to function as a downstream mediator of the transforming activity of oncogenic Ha-Ras and c-Myc 27, to promote proliferation via suppression of FOXO1, as well as to suppress apoptosis and increase anchorage-independent growth of non-tumorigenic astrocytes by activating PI3K-Akt and NF- $\kappa B$ pathways (28-30). These studies suggest that AEG-1 overexpression plays a dominant positive role in the progression of numerous types of cancer. However, the biological significance of AEG-1 in cervical carcinoma has not been fully elucidated yet.

In the present study, we demonstrated that the expression of AEG-1 was upregulated in 3 cervical carcinoma cell lines 
as well as in cervical carcinoma tissue (CCT) specimens, and that overexpression of AEG-1 was correlated with the clinical characteristics of the disease. Moreover, we also showed that AEG-1 overexpression promoted angiogenesis through the increased expression of the angiogenesis-related genes $H I F-1 \alpha, T i e 2, V E G F$ and TEMI/CD248. Our results suggest that AEG-1 constitutes a novel and valuable predictive factor for the prognostic evaluation of cervical carcinoma patients.

\section{Materials and methods}

CCT specimens and cell culture. The 3 human cervical carcinoma cell lines HeLa, CaSki and SiHa were purchased from the American Type Culture Collection (ATCC) and cultured in complete RPMI-1640 medium (Gibco, Grand Island, NY, USA). Normal cervical epithelial cells (NCECs) and human umbilical vein endothelial cells (HUVECs) were established and maintained in our laboratory $(31,32)$. Eight paired primary CCT specimens and matched normal tissues obtained from the same patients were collected at the Department of Gynecology and Obstetrics of Tangdu Hospital (Fourth Military Medical University, Xi'an, China). Tissue arrays containing 200 paraffin-embedded archival CCT specimens and 8 normal cervical tissues (nos. CR208-1 and CR208-3) were purchased from Chaoying Biotech Company (Xian, China). Patient informed consent and approval from the Institutional Research Ethics Committee of Tangdu Hospital were obtained prior to use of the clinical material for research purposes. Patient clinicopathological characteristics are provided in Table I.

Immunohistochemical analysis (IHC). IHC was performed as previously described (31) in 200 human CCT and 8 normal cervical tissue specimens. Rabbit anti-human AEG-1 polyclonal antibody (Proteintech Group, Chicago, IL, USA) was used as a primary antibody and goat anti-rabbit IgG coupled to horseradish peroxidase (HRP) (Santa Cruz Biotechnology, Inc., Santa Cruz, CA, USA) was used as a secondary antibody. Immunostaining was observed in 5 areas of each slide and scored independently by two observers, based on both the proportion of positively stained tumor cells and the intensity of staining. The proportion of positive cells was scored as follows: 0 , no positive cells; $1,<10 \%$ positive cells; $2,10-50 \%$ positive cells; and $3,>50 \%$ positive cells. The intensity of staining was stratified into 4 categories: 0 , no staining; 1 , weak staining (light yellow); 2, moderate staining (yellow brown); and 3, strong staining (brown). The staining index (SI) was calculated as follows: Staining intensity score $\mathrm{x}$ proportion of positive tumor cells. Using this method of assessment, we evaluated the expression of AEG-1 in 200 archival CCT specimens by determining SI which was scored as 0, 1, 2, 3, 4, 6 and 9. An SI score of 0 was marked as '-', 1 and 2 as ' + ', 3 and 4 as '++', 6 and 9 as '+++'.

Vector construction and cell transfection. shRNA expression vectors were generated by annealing single-stranded oligonucleotides and inserting them into the BamHI and HindIII enzyme sites of pSilencer4.1-CMVneo vector (Ambion, Austin, TX, USA). The target sequences were as follows: shA1 (AEG-1; GenBank, AF411226.1; 1825-1843 bp): 5'-GTGCCG
Table I. Patient clinicopathological characteristics.

\begin{tabular}{lc}
\hline Characteristic & No. of cases $(\%)$ \\
\hline Age (years) & \\
$\leq 50$ & $115(57.5)$ \\
$>50$ & $85(42.5)$ \\
Clinical stage & \\
I & $35(17.5)$ \\
II & $130(65)$ \\
III & $25(12.5)$ \\
IV & $10(5)$
\end{tabular}

Tumor differentiation

G1

$\mathrm{G} 2$

G3

T classification

$\mathrm{T} 1$

$\mathrm{T} 2$

$\mathrm{T} 4$

N1

M classification (distant metastasis)

M0

CCAATACTACAAG-3' (recommended by P.B. Fisher, Departments of Pathology and Urology, Columbia University, USA); shA2 (AEG-1; GenBank, AF411226.1; 666-686 bp): AACAGAAGAAGAAGAACCGGA (27); and a scrambled sequence was used as a negative control (NC): 5'-TTCTCC GAACGTGTCACGT-3' (provided by Ambion). The recombinant shRNA vectors were named pshA1, pshA2 and pshNC. The full-length open reading frame cDNA of $A E G-1$ was amplified using RT-PCR from total mRNA of HeLa cells, and then inserted into the pcDNA3.1 expression vector; the recombinant vector was named pAEG1. All recombinant vectors were confirmed by enzyme digestion and DNA sequencing analysis. Cell transfection was performed using Lipofectamine $^{\mathrm{TM}} 2000$ (Invitrogen, Carlsbad, CA, USA) following the manufacturer's instructions and selected with $600 \mu \mathrm{g} / \mathrm{ml}$ of G418 (Invitrogen, San Diego, CA, USA) after transfection.

RT-PCR and quantitative real-time RT-PCR. RT-PCR was conducted as previously described (33). Briefly, total RNA was extracted using the TRIzol reagent (Invitrogen, Carlsbad, CA, USA), and $1 \mu \mathrm{g}$ RNA was used to synthesize cDNA using the M-MLV reverse transcriptase kit (Takara Biotechnology, Dalian, China) according to the manufacturer's protocol. The cDNA was then used to amplify the mRNA fragment. $\beta$-actin was also amplified as an internal standard. The corresponding primer sequences for RT-PCR amplification are provided in 
Table II. Primer pairs used in RT-PCR and real-time RT-PCR.

\begin{tabular}{|c|c|c|c|}
\hline & Gene & GenBank ID & Primer pairs $\left(5^{\prime} \rightarrow 3^{\prime}\right)$ \\
\hline \multirow[t]{6}{*}{ RT-PCR } & $A E G-1$ & AF411226 & $\begin{array}{l}\text { F: GTGAAGCTGTTCGAACACCTCAAAG } \\
\text { R: GACAGTGAGGTTTTCATTCAATCCTG }\end{array}$ \\
\hline & TEMI & NM_020404 & $\begin{array}{l}\text { F: TCGAGTGTTATTGTAGCGAGGGACATG } \\
\text { R: AGGTGGGCTCCGGGTAGGGTAT }\end{array}$ \\
\hline & $H I F-1 \alpha$ & NM_181054 & $\begin{array}{l}\text { F: GCAAGACTTTCCTCAGTCGACACA } \\
\text { R: GCATCCTGTACTGTCCTGTGGTGA }\end{array}$ \\
\hline & Tie2 & NM_000459 & $\begin{array}{l}\text { F: GCTGTCATCAACATCAGCTCTG } \\
\text { R: GAGGAGGGAGTCCGATAGAAGC }\end{array}$ \\
\hline & $V E G F$ & NM_001025366 & $\begin{array}{l}\text { F: ATGGCAGAAGGAGGAGGG } \\
\text { R: TTGGACTCCTCAGTGGGC }\end{array}$ \\
\hline & $\beta$-actin & NM_001101.3 & $\begin{array}{l}\text { F: GACTTAGTTGCGTTACACCCTTTC } \\
\text { R: TGCTGTCACCTTCACCGTTC }\end{array}$ \\
\hline \multirow[t]{2}{*}{ Real-time RT-PCR } & $A E G-1$ & AF411226 & $\begin{array}{l}\text { F: GGGGAAGGAGTTGGAGTGAC } \\
\text { R: GTAGACTGAGAAACTGGCTCAGCAG }\end{array}$ \\
\hline & GAPDH & NG 007073.2 & $\begin{array}{l}\text { F: CCACATCGCTCAGACACCAT } \\
\text { R: GGCAACAATATCCACTTTACCAGAGT }\end{array}$ \\
\hline
\end{tabular}

Table II. The RT-PCR products were then electrophoresed through a $1.5 \%$ agarose gel, and signals were quantified by densitometric analysis using Multilmage ${ }^{\mathrm{TM}}$ Light Cabinet (Alpha Innotech Corporation, San Leandro, CA, USA).

Real-time RT-PCR was performed using ABI 7500 Real-Time RT-PCR system and SYBR Premix Ex Taq II for individual mRNAs according to the manufacturer's protocol (Takara Biotechnology). Primer pairs are shown in Table II. Expression data were normalized to the geometric mean of the $G A P D H$ gene to control the variability in expression levels.

Western blot analysis. Protein levels were determined by western blot analysis as previously described (33). Target proteins were detected using specific antibodies against AEG-1 (Proteintech, Chicago, IL, USA); TEM1 (Abcam, Cambridge, UK); Tie2, HIF-1 $\alpha$ and VEGF (Santa Cruz Biotechnology, Inc.). All secondary antibodies of IgG coupled to HRP were purchased from Santa Cruz Biotechnology, Inc. Densitometric analysis was performed by photoimage analysis using the Multilmage $^{\mathrm{TM}}$ Light Cabinet. $\beta$-actin was used as a loading control and the results are expressed as the protein $/ \beta$-actin absorbance ratio.

Flow cytometric analysis. For cell cycle analysis, the cells were fixed with $70 \%$ ethanol and stained with propidium iodide (PI). Cell cycle distribution was then analyzed on a FACSCalibur system (BD Biosciences, Bedford, MA, USA) using ModFit software (Verity Software House, Topsham, ME, USA). For apoptosis detection, the cells were pelleted, suspended in Annexin V-fluorescein isothiocyanate (FITC) $(0.5 \mathrm{mg} / \mathrm{ml})$ and PI $(0.6 \mathrm{mg} / \mathrm{ml})$, and then analyzed with WinMDI software (The Scripps Research Institute, La Jolla, CA, USA) using FACSCalibur system.
Scratch wound migration assay. The cells were seeded onto 6-well plates and grown until they reached $90 \%$ confluence. A linear wound was then created in the confluent monolayer using a pipette tip. The wounded monolayer was washed to remove cell debris, and wounds were then observed and photographed at various indicated times. The migrated cells were analyzed under a light microscope. Each experiment was repeated three times.

Tube formation assay. Matrigel (BD Biosciences) was dissolved at $4^{\circ} \mathrm{C}$ overnight and 96-well plates were prepared with $55 \mu 1$ Matrigel in each well and incubated at $37^{\circ} \mathrm{C}$ for 30 min. HUVECs were seeded on coated plates at a density of $2 \times 10^{4}$ cells/well in Dulbecco's modified Eagle's medium (DMEM) containing 2\% fetal bovine serum (FBS). The culture media of HUVECs were replaced with the media from HeLa cells, pshAEG1-transfected HeLa cells, NCECs and pAEG1-transfected NCECs, and then all treated HUVECs were incubated at $37^{\circ} \mathrm{C}$ overnight. Five different fields were randomly chosen in each well and images were captured. The length of the tubes was measured using Olympus DP2-BSW software (Soft Imaging System GmbH, Münster, Germany) and was expressed as total length (mm) per microscopic field for each well.

Statistical analysis. Results are expressed as means \pm standard deviation (SD). Statistical analyses were performed using SPSS version 17.0 (IBM Company, Chicago, IL, USA). The Mann-Whitney U test and the Kruskal-Wallis H test were used to analyze the correlation between AEG-1 expression and clinicopathologic characteristics. $\mathrm{P}<0.05$ was considered to indicate a statistically significant difference which is indicated by letters or asterisks, respectively, in the tables and figures. 
Table III. Correlation of AEG-1 expression with the clinicopathological characteristics of 200 cervical carcinoma specimens.

\begin{tabular}{|c|c|c|c|c|c|}
\hline \multirow[b]{2}{*}{ Characteristic } & \multicolumn{5}{|c|}{ Expression of AEG-1 protein } \\
\hline & $\begin{array}{l}\text { Negative }(-) \\
(\mathrm{n}=20), \mathrm{n}(\%)\end{array}$ & $\begin{array}{l}\text { Positive (+) } \\
(\mathrm{n}=30), \mathrm{n}(\%)\end{array}$ & $\begin{array}{l}\text { Positive }(++) \\
(\mathrm{n}=68), \mathrm{n}(\%)\end{array}$ & $\begin{array}{l}\text { Positive }(+++) \\
(\mathrm{n}=82), \mathrm{n}(\%)\end{array}$ & P-value \\
\hline \multicolumn{6}{|l|}{ Age (years) } \\
\hline$\leq 50$ & $15(75)$ & $17(56.7)$ & $37(54.4)$ & $46(56.1)$ & \multirow[t]{2}{*}{0.248} \\
\hline$>50$ & $5(25)$ & $13(43.3)$ & $31(45.6)$ & $36(43.9)$ & \\
\hline \multicolumn{6}{|l|}{ Clinical stage } \\
\hline I & $10(50)$ & $5(16.7)$ & $15(22.1)$ & $5(6.1)$ & \multirow[t]{4}{*}{$0.034^{\mathrm{a}}$} \\
\hline II & $10(50)$ & $25(83.3)$ & $50(73.5)$ & $45(54.9)$ & \\
\hline III & $0 \quad(0)$ & $0(0)$ & 3 (4.4) & $22(26.8)$ & \\
\hline IV & 0 & $0(0)$ & $0 \quad(0)$ & $10(12.2)$ & \\
\hline \multicolumn{6}{|c|}{ Tumor differentiation } \\
\hline G1 & 0 & $5(16.7)$ & $10(14.7)$ & $21(25.6)$ & \multirow[t]{3}{*}{$0.043^{\mathrm{a}}$} \\
\hline G2 & $11(55)$ & $20(66.7)$ & $56(82.4)$ & $61(74.4)$ & \\
\hline G3 & $9(45)$ & $5(16.7)$ & $2(2.9)$ & $0 \quad(0)$ & \\
\hline \multicolumn{6}{|l|}{$\mathrm{T}$ classification } \\
\hline $\mathrm{T} 1$ & $17(85)$ & $27(90)$ & $47(69.1)$ & $33(40.2)$ & \multirow[t]{4}{*}{$0.019^{\mathrm{a}}$} \\
\hline $\mathrm{T} 2$ & $3(15)$ & $3(10)$ & $21(30.9)$ & $38(46.3)$ & \\
\hline $\mathrm{T} 3$ & $0 \quad(0)$ & $0 \quad(0)$ & $0(0)$ & $6(7.3)$ & \\
\hline $\mathrm{T} 4$ & $0 \quad(0)$ & $0 \quad(0)$ & $0(0)$ & $5(6.1)$ & \\
\hline \multicolumn{6}{|l|}{$\mathrm{N}$ classification } \\
\hline N0 & $20(100)$ & $30(100)$ & $68(100)$ & $59(71.9)$ & \multirow[t]{2}{*}{$0.038^{\mathrm{a}}$} \\
\hline N1 & 0 & $0 \quad(0)$ & $0 \quad(0)$ & $23(28.1)$ & \\
\hline \multicolumn{6}{|c|}{ M classification (distant metastasis) } \\
\hline M0 & $20(100)$ & $30(100)$ & $68(100)$ & $73(89)$ & \multirow[t]{2}{*}{$0.018^{\mathrm{a}}$} \\
\hline M1 & $\begin{array}{ll}0 & (0)\end{array}$ & $0 \quad(0)$ & $\begin{array}{ll}0 & (0)\end{array}$ & $9(11)$ & \\
\hline
\end{tabular}

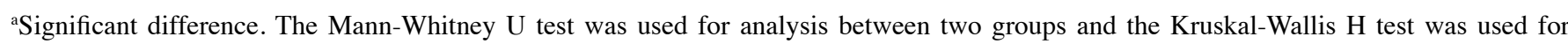
analysis among three or up to 3 groups. G1, weak differentiation; G2, moderate differentiation; G3, high differentiation.

\section{Results}

AEG-1 expression is upregulated in cervical carcinoma cells and primary CCT specimens. To determine AEG-1 protein and mRNA expression levels in cervical carcinomas, we first performed semi-quantitative RT-PCR and western blot analysis in the following cell lines: SiHa, HeLa and CaSki. RT-PCR results revealed that AEG-1 mRNA was overexpressed in all the 3 cervical carcinoma cell lines, while it was weakly detected in NCECs (Fig. 1A). In parallel with the upregulated AEG-1 mRNA expression, western blot analysis showed that all cervical carcinoma cell lines exhibited significantly higher levels of AEG-1 protein compared with that of the NCECs (Fig. 1B, P<0.05). Furthermore, real-time RT-PCR analysis of 8 cases of paired primary CCT and adjacent non-cancerous tissue specimens demonstrated that the levels of AEG-1 expression in all 8 cervical CCT specimens were found to be obviously upregulated compared with their matched adjacent non-cancerous tissues (Fig. 1C). These results demonstrate that AEG-1 is overexpressed in CCTs which is suggested to be primarily caused by transcriptional upregulation.
A
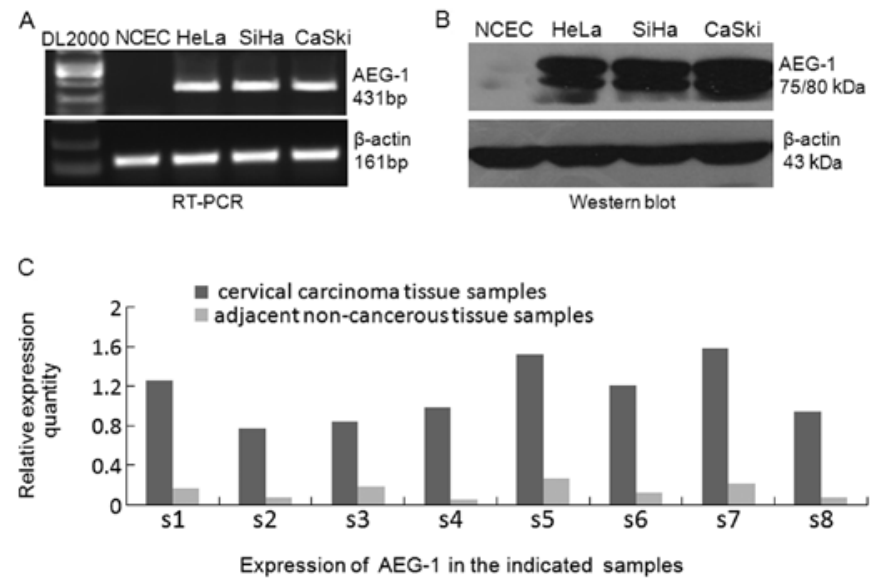

Figure 1. AEG-1 is overexpressed in primary CCT specimens and cervical carcinoma cell lines. (A) RT-PCR analysis of $A E G-1$ expression in 3 cervical carcinoma cell lines. $\beta$-actin was used as a loading control. (B) Western blot analysis of AEG-1 protein expression in 3 cervical carcinoma cell lines. (C) Real-time RT-PCR analysis of $A E G-1$ expression in 8 paired primary CCT specimens (s1-8) and their adjacent non-cancerous tissues. The expression levels were normalized for GAPDH. All experiments were repeated thrice. NCEC, normal cervical epithelial cells. 


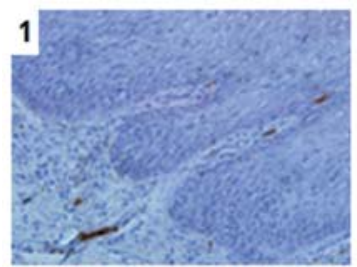

Normal cervical tissue

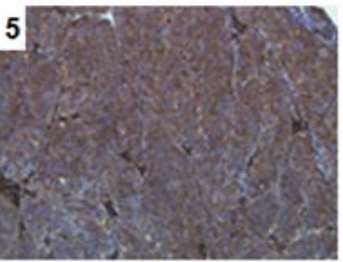

Squamous cell carcinoma Grade II, T1a1N1M0

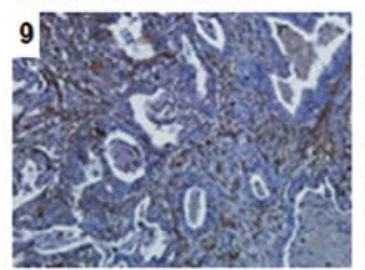

Adenocarcinoma Grade II , T1b2NOMO

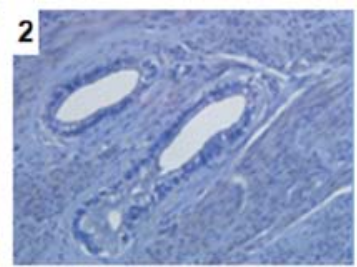

Cancer adjacent normal cervix tissue

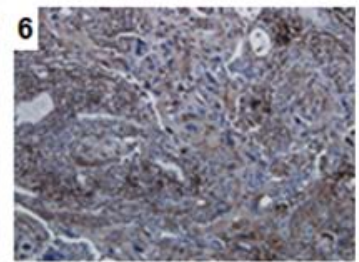

Squamous cell carcinoma Grade III, T2aNOMO

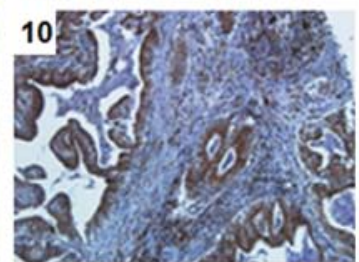

Endometrioid adenocarcinoma, T1a1 NOMO

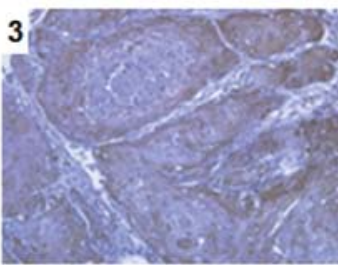

Squamous cell carcinoma Grade I, T1NOMO

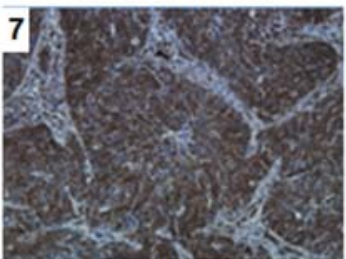

Squamous cell carcinoma Grade III, T2aN1M1

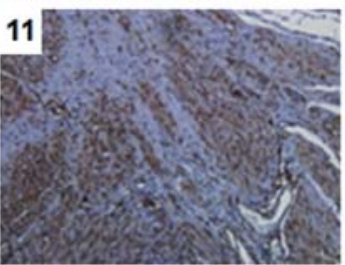

Endocervical-type adenocarcinoma, Grade I, T1b1 NOMO

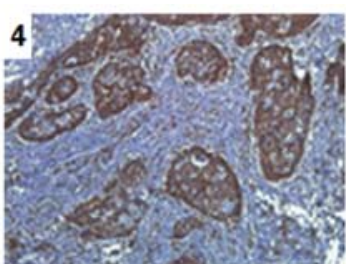

Squamous cell carcinoma

Grade II , T1NOMO

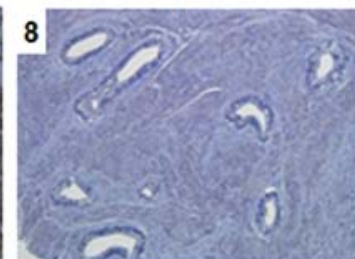

Adenocarcinoma

Grade I , T1bNOMO

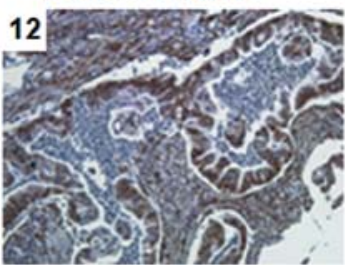

Endocervical-type

adenocarcinoma,

Grade II , T2bNOMO

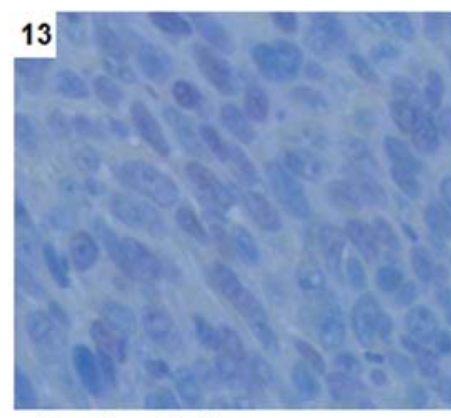

Normal cervical tissue

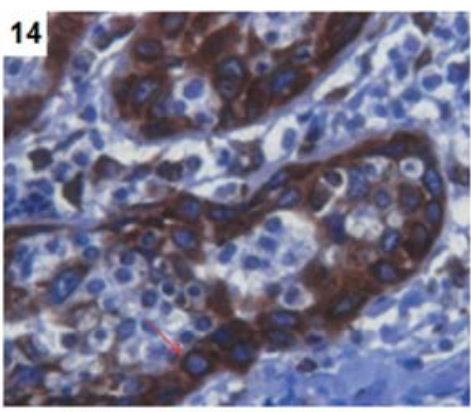

Squamous cell carcinoma Grade II , T1NOMO

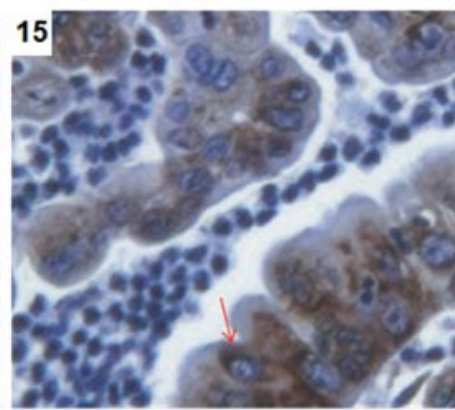

Endocervical-type adenocarcinoma Grade II , T2bNOMO

Figure 2. AEG-1 protein is overexpressed in archived cervical carcinoma tissue (CCT) specimens. Representative images of 200 archived CCT and 8 normal cervical epithelial tissue specimens following immunohistochemical (IHC) staining. AEG-1 staining was mainly localized in the cytoplasm of primary cancer cells. (1 and 2) Expression of AEG-1 in normal cervical tissue and cancer adjacent tissue. (3-12) Expression of AEG-1 in pathological CCT lesions of different clinical stages (magnification, x100). (13-15) Expression of AEG-1 in normal cervical tissue and pathological CCT lesions of different clinical stages (magnification, x400). Red arrows indicate positively stained cells (images 14 and 15).

Overexpression of AEG-1 in archived CCT specimens is associated with clinical staging of cervical carcinoma patients. To further elucidate whether AEG-1 overexpression is associated with clinicopathological characteristics of cervical carcinoma, 200 archived CCT and 8 normal cervical tissue specimens were examined by IHC staining with anti-AEG-1 antibody. As shown in Fig. 2, AEG-1 protein was detected in 180/200 (90\%) cases. By contrast, weak or negative signals were observed in normal cervical tissues. Statistical analysis revealed that the expression of AEG-1 was strongly correlated with the clinical staging of cervical carcinoma patients $(\mathrm{P}=0.034)$ and tumor differentiation $(\mathrm{P}=0.043)$. Furthermore, significant differences were also found in AEG-1 expression in patients categorized according to $\mathrm{T}(\mathrm{P}=0.019), \mathrm{N}(\mathrm{P}=0.038)$ and $\mathrm{M}$ classification $(\mathrm{P}=0.018)$, indicating that advanced clinical stages and $\mathrm{T}$ classification were correlated with higher AEG-1 expres- sion (Table III). However, there were no obvious correlations between the levels of AEG-1 protein expression and patient age. These results showed that higher clinical stage and T classification or metastasis were correlated with a higher level of AEG-1 protein expression.

Knockdown of AEG-1 inhibits proliferation and promotes the apoptosis of cervical carcinoma cells. To determine the biological role of AEG-1 overexpression in cervical carcinoma, we transfected the AEG-1 shRNA expression vectors pshA1 and pshA2 into SiHa cells, and examined the cell growth and apoptosis by flow cytometry. As shown in Fig. 3A, both pshA1 and pshA2 vectors effectively knocked down AEG-1 expression in SiHa cells, which was followed by a subsequent decrease in cell proliferation by 39.95 and $22.39 \%$, respectively, at $72 \mathrm{~h}$ compared with the control 

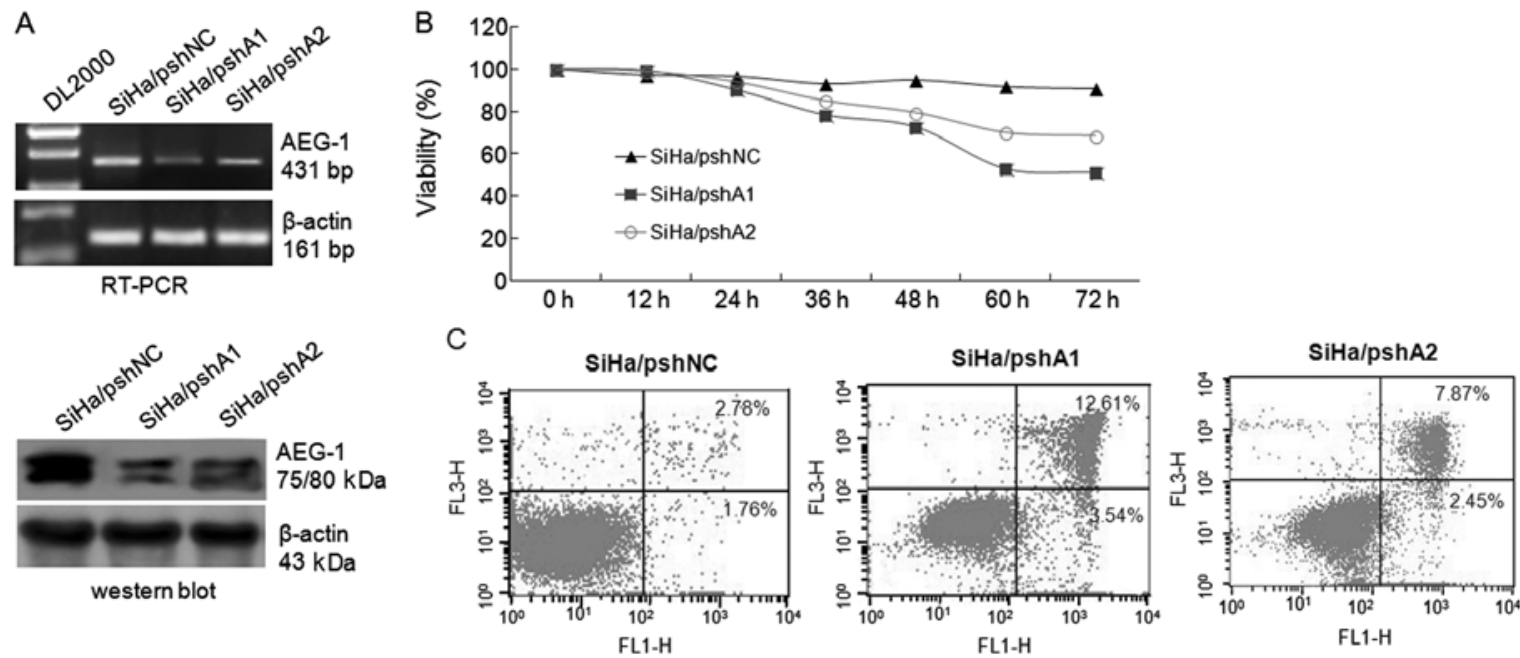

Figure 3. Knockdown of AEG-1 inhibits proliferation and promotes apoptosis in cervical carcinoma cells. (A) RT-PCR and western blot analysis of AEG-1 expression in the indicated cells. Transfection with pshA1 and pshA2 effectively inhibited AEG-1 expression at the mRNA and protein levels. $\beta$-actin was used as a loading control. (B) Cell proliferation analysis in the indicated cells using the MTT assay. (C) Flow cytometric analysis of apoptosis showing increased apoptotic rates of pshA1- and pshA2-transfected $\mathrm{SiHa}$ cells $(\mathrm{P}<0.05$, respectively). Each experiment was repeated thrice.

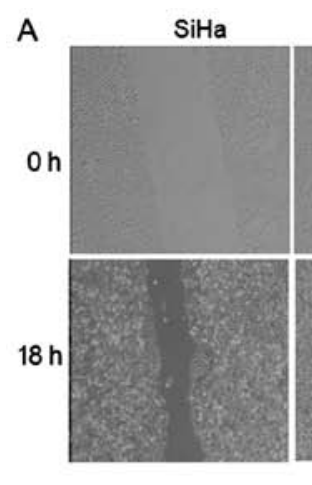

B

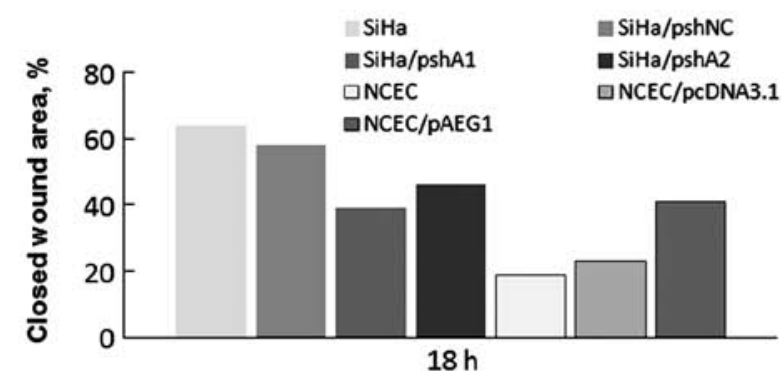

$\mathrm{SiHa/pshNC}$
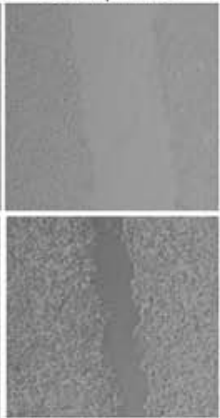
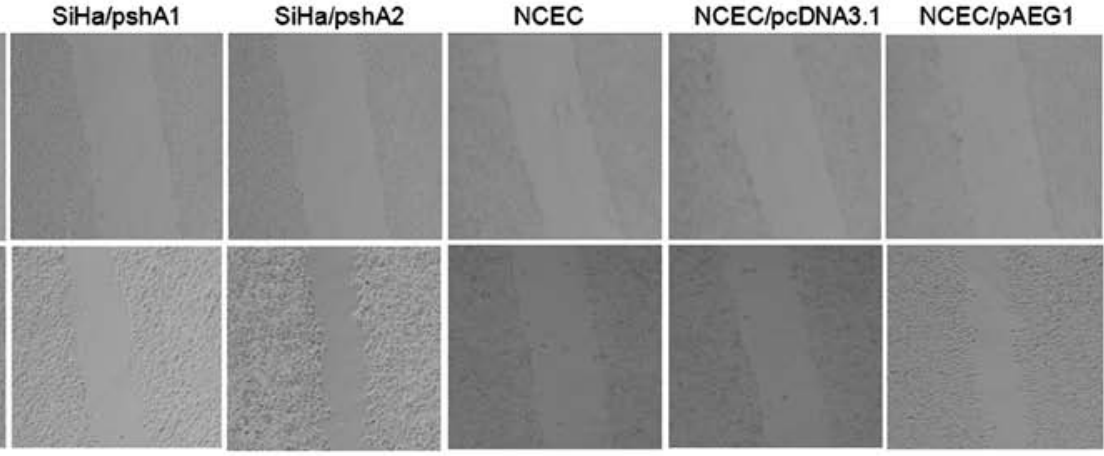

C

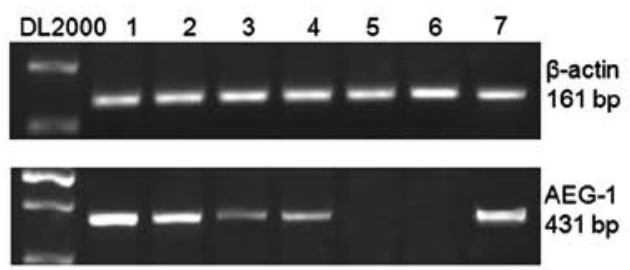

Figure 4. Knockdown of AEG-1 expression in SiHa cells reduced cell migration ability. (A) Representative wound healing images at 0 and 18 h. Wounds were constructed with a pipette tip in confluent monolayers. (B) Quantification of wound healing rates. Data are means \pm SD. (C) RT-PCR detection of AEG-1 expression in the indicated cells during the wound healing assay. Each experiment was repeated thrice. Lane 1, SiHa; lane 2, SiHa/pshNC; lane 3, SiHa/pshA1; lane 4, SiHa/pshA2; lane 5, NCEC; lane 6, NCEC/pcDNA3.1; lane 7, NCEC/pAEG1. NCEC, normal cervical epithelial cells.

cells (Fig. 3B). Furthermore, analysis of apoptosis showed that the apoptotic rate of pshA1-transfected SiHa cells was significantly increased to $16.15 \pm 4.6$ and $10.32 \pm 3.4 \%$ when compared with the pshNC-transfected control cells (Fig. 3C, $\mathrm{P}<0.05)$. These results demonstrate that AEG-1 overexpression in cervical carcinoma cells plays an important role in cell proliferation and apoptosis, and that downregulation of AEG-1 expression not only leads to inhibition of cervical carcinoma cell proliferation, but also promotes tumor cell apoptosis.
Knockdown of AEG-1 reduces the migration ability of cervical carcinoma cells. It was previously shown that AEG-1 promotes the invasive ability of various types of cancer cells $(11,18)$. To further investigate the role of AEG-1 overexpression in the migratory ability of cervical carcinoma cells, a scratch wound migration assay was conducted. As shown in Fig. 4A, the overexpression of AEG-1 in NCECs led to a significantly increased invasive ability compared with the ability of the control vector-transfected NCECs. Conversely, silencing of AEG-1 expression in SiHa cells inhibited the migratory speed 
A

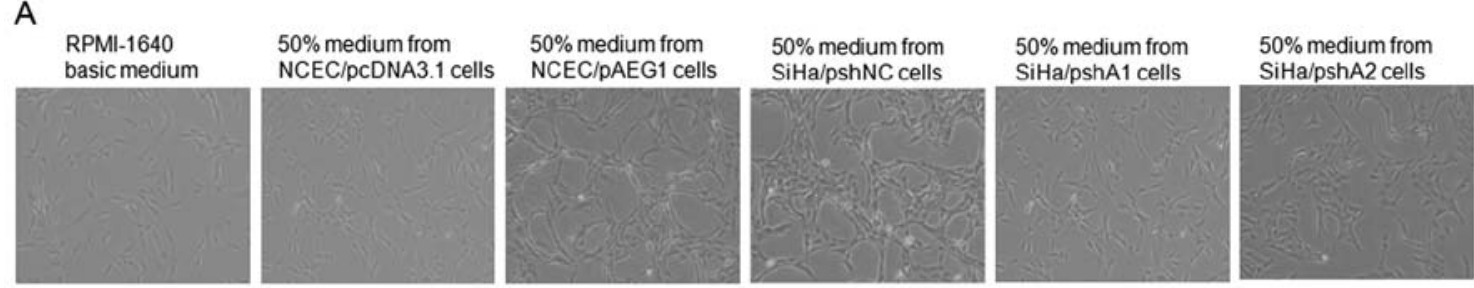

B
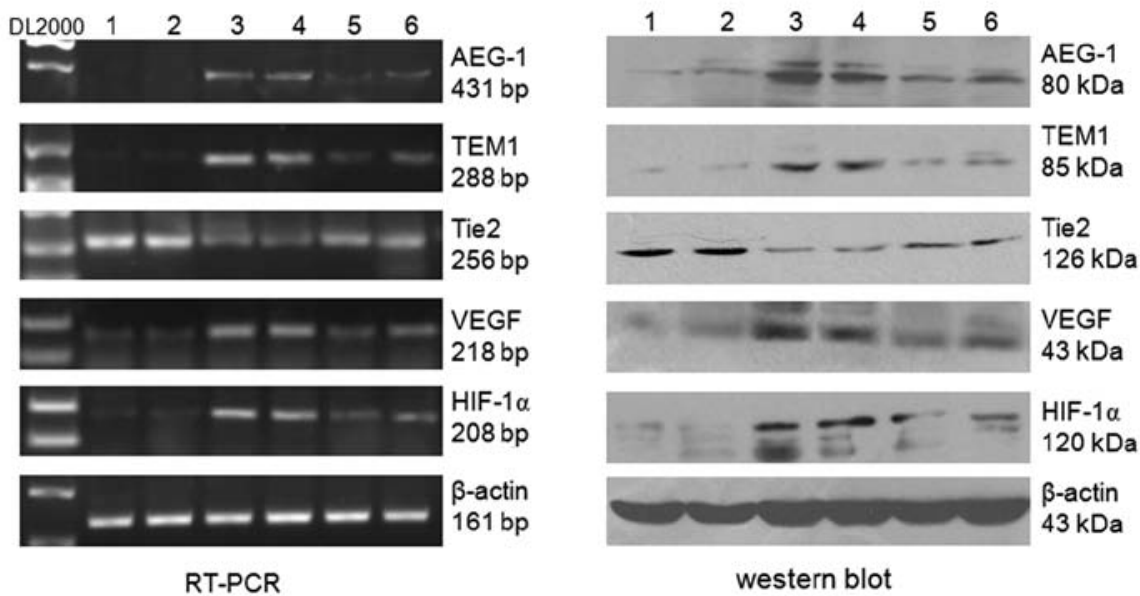

Figure 5. AEG-1 expression influences the migration and tube formation of HUVECs. (A) HUVECs were treated with conditioned media from the indicated cells for $24 \mathrm{~h}$ and tube formation was photographed. (B) RT-PCR and western blot analysis of angiogenesis-associated genes and their protein expression in HUVECs after treatment with the conditioned media. The levels of expression were normalized with $\beta$-actin levels in each sample. This experiment was repeated thrice. Lane 1, HUVECs treated with RPMI-1640 basic medium; lane 2, HUVECs treated with 50\% medium from NCEC/pcDNA3.1 cells; lane 3 , HUVECs treated with 50\% medium from NCEC/pAEG1 cells; lane 4, HUVECs treated with 50\% medium from SiHa/pshNC cells; lane 5, HUVECs treated with 50\% medium from SiHa/pshA1 cells; lane 6, HUVECs treated with 50\% medium from SiHa/pshA2 cells. HUVECs, human umbilical vein endothelial cells; NCEC, normal cervical epithelial cells.

and reduced the invasive ability of the cells. Meanwhile, the expression of AEG-1 in the cells that were used during the wound healing assay was detected using RT-PCR as shown in Fig. 4C. These results confirm the role of AEG-1 in regulating the invasive ability of cervical carcinoma cells.

AEG-1 expression influences the migration and tube formation ability of HUVECs through several angiogenesis-associated molecular markers. The role of AEG-1 deregulation both in the angiogenesis of tumor cells and HUVECs was examined in vitro. We first assessed whether downregulation of AEG-1 in SiHa cells influences the tube formation ability of HUVECs by co-culture in conditioned medium. As shown in Fig. 5A, the medium from $\mathrm{SiHa}$ and $\mathrm{SiHa}$ cells transfected with the control vector ( $\mathrm{SiHa} / \mathrm{pshNC}$ ) significantly promoted the tube formation ability of HUVECs, while the medium from AEG-1-depleted SiHa cells ( $\mathrm{SiHa} / \mathrm{pshA} 1$ and $\mathrm{SiHa} / \mathrm{pshA2}$ ) showed no obvious effect on the tube formation ability of HUVECs when compared with the medium from control cells. To further investigate the role of the upregulation of AEG-1 in angiogenesis, the full-length AEG-1 expression vector pAEG1 was transfected into NCECs, and the results from co-culture in conditioned medium showed that ectopic expression of AEG-1 in NCECs significantly increased the tube formation ability of HUVECs when compared with the control cells (Fig. 5A). RT-PCR results revealed that co-culture of HUVECs with the medium from $\mathrm{SiHa} / \mathrm{pshNC}$ and NCEC/pAEG1 cells significantly decreased the expression level of Tie 2 and increased the expression levels of HIF-1 $\alpha$, VEGF and TEM1/CD248 (Fig. 5B). These results demonstrate that overexpression of AEG-1 in HeLa cells plays a dominant positive role in regulating oncogenic angiogenesis. To the best of our knowledge, this is the first report that AEG-1 promotes the expression of TEM1/CD248.

\section{Discussion}

Cervical carcinoma is the third most common type of cancer among women worldwide, and the majority of new cases present with advanced stages (34) potentially due to the limited access to screening programs. Many diagnostic markers of cervical carcinoma and markers for disease follow-up have been identified and investigated; however, a useful screening marker of cervical carcinoma has not yet been clearly established. Thus, the identification of novel molecular prognostic and predictive markers of cervical carcinoma is suggested to support the accurate evaluation of patient prognosis and the proper stratification of patients into different risk groups. This would subsequently lead to the administration of specific adjuvant therapies and would, finally, increase patient survival time.

Recently, numerous studies have demonstrated that AEG-1 is upregulated in various types of cancers, and that it is correlated with cancer progression and patient prognosis $(10,13,14,16,18)$. The present study was performed in order to investigate whether AEG-1 plays a role in cervical 
carcinoma. We showed that AEG-1 is upregulated at both the mRNA and protein levels in cervical carcinoma cells when compared with NCECs. Paired CCT and adjacent non-cancerous tissues were also found to differentially express AEG-1, with the cancer tissues displaying a significantly higher expression of AEG-1 (Fig. 1). Considering the oncogenic properties of AEG-1 protein and its ability to promote the proliferation of various cell types in vitro, the high expression of AEG-1 protein observed in cervical carcinoma cells and tumor tissues suggests that AEG-1 is involved in the progression of human cervical carcinoma in vivo. Thus, we analyzed the correlation between AEG-1 expression and the clinical characteristics of cervical carcinoma patients. IHC staining (Fig. 2) showed that the expression level of AEG-1 protein in histological sections was significantly correlated with the clinical stage of the disease of patients with cervical carcinoma. These results demonstrated that AEG-1 plays an important role in the pathogenesis of cervical carcinoma and that it represents a novel prognostic indicator for cervical carcinoma.

Based on the above-mentioned findings, we further investigated the biological roles of AEG-1 by loss- and gain-of-function analyses to determine the potential use of AEG-1 as a therapeutic target for the treatment of cervical carcinoma. As shown in Fig. 3B, knockdown of AEG-1 led to a significant inhibition of cell proliferation in vitro. Moreover, significantly increased rates of cell apoptosis were detected in the stably pshA1-transfected cervical carcinoma cells. Moreover, silencing of AEG-1 expression obviously inhibited the migratory ability of cervical carcinoma cells. Conversely, ectopic expression of AEG-1 in NCECs led to a significantly increased invasive ability compared with the control vector-transfected cells (Fig. 4). These results confirmed that AEG-1 plays an important role in the pathogenesis of cervical carcinoma through modulation of the cell cycle, apoptosis and invasive ability of the cells. Thus, AEG-1 is suggested to constitute a therapeutic target for the treatment of cervical carcinoma.

Increasing evidence has shown that AEG-1-expressing tumors have increased microvessel density (MVD) and that AEG-1 overexpression promotes angiogenesis both in vitro and in vivo, suggesting that AEG-1 plays an important role in tumor angiogenesis $(23,27)$. In the present study, co-culture with conditioned medium was performed to assess the influence of the downregulation or upregulation of AEG-1 expression in SiHa cells or NCECs on the tube formation ability of HUVECs. The results showed that the medium from $\mathrm{SiHa}$ or $\mathrm{SiHa} / \mathrm{pshNC}$ cells significantly promoted the tube formation ability of HUVECs, while the medium from AEG-1-depleted SiHa cells showed no obvious effect on the tube formation ability of HUVECs when compared with that from the control cells (Fig. 5A). These results demonstrated that aberrant expression of AEG-1 caused cervical carcinoma cells to secrete angiogenesis-associated cytokines which promoted the tube formation ability of HUVECs. Angiogenesis occurs during the early stages of cancer development and is essential for the advancement of solid tumors (35). Meanwhile, medium from NCECs transfected with full-length AEG-1 cDNA of the pAEG1 vector led to a significant increase in the tube formation ability of HUVECs when compared with that of the control cells. In addition, ectopic expression of AEG-1 in HUVECs induced the expression of several angiogenesis-associated molecular markers, including HIF-1 $\alpha$, Tie2, VEGF and TEM1/CD248 (Fig. 5B). To the best of our knowledge, this is the first report that TEM1/CD248 is an AEG-1-induced angiogenesis marker; however, the effects on angiogenesis and the role of TEM1/CD28 as a predictive factor for the prognostic evaluation of cervical carcinoma need to be further investigated. These results confirmed that overexpression of AEG-1 in SiHa cells plays an important role in the regulation of oncogenic angiogenesis.

In conclusion, our data suggest that AEG-1 is an important mediator of angiogenesis and a valuable prognostic factor in patients with cervical carcinoma. AEG-1 protein is also suggested to provide a therapeutic target for the treatment of cervical carcinoma.

\section{Acknowledgements}

This study was supported by a grant from the National Natural Science Foundation of China (no. 81201525). We thank all staff members of the Department of Medical Laboratory and the Research Center in Tangdu Hospital of the Fourth Military Medical University (Xi'an, China) for their sincere help and technical support. We also thank all staff members of the Department of Gynecology and Obstetrics of the Tangdu Hospital of the Fourth Military Medical University for kindly providing patient samples.

\section{References}

1. Jemal A, Bray F, Center MM, Ferlay J, Ward E and Forman D: Global cancer statistics. CA Cancer J Clin 61: 69-90, 2011.

2. Green JA, Kirwan JM, Tierney JF, et al: Survival and recurrence after concomitant chemotherapy and radiotherapy for cancer of the uterine cervix: a systematic review and meta-analysis. Lancet 358: 781-786, 2001.

3. Martin-Loeches M, Ortí RM, Cazorla E, Asins E and Lixiona J: Multivariate analysis of the morphometric characteristics of tumours as prognostic factors in the survival of patients with uterine cervix cancer treated with radical surgery. Eur J Obstet Gynecol Reprod Biol 105: 170-176, 2002.

4. Gasinska A, Urbanski K, Adamczyk A, Pudelek J, Lind BK and Brahme A: Prognostic significance of intratumour microvessel density and haemoglobin level in carcinoma of the uterine cervix. Acta Oncol 41: 437-443, 2002.

5. Goudy G, Stoeckle E, Thomas L, et al: Prognostic impact of tumour volume and lymph node involvement in intermediate stage T1b1 to T2b cancer of the uterine cervix. Bull Cancer 96: 685-694, 2009 (In French).

6. Grigiene R, Valuckas KP, Aleknavicius E, Kurtinaitis J and Letautiene SR: The value of prognostic factors for uterine cervical cancer patients treated with irradiation alone. BMC Cancer 7: 234, 2007.

7. Su ZZ, Kang DC, Chen Y, et al: Identification and cloning of human astrocyte genes displaying elevated expression after infection with HIV-1 or exposure to HIV-1 envelope glycoprotein by rapid subtraction hybridization, RaSH. Oncogene 21: 3592-3602, 2002.

8. Brown DM and Ruoslahti E: Metadherin, a cell surface protein in breast tumors that mediates lung metastasis. Cancer Cell 5: 365-374, 2004.

9. Sarkar D, Emdad L, Lee SG, Yoo BK, Su ZZ and Fisher PB: Astrocyte elevated gene-1: far more than just a gene regulated in astrocytes. Cancer Res 69: 8529-8535, 2009.

10. Liao WT, Guo L, Zhong Y, Wu YH, Li J and Song B: Astrocyte elevated gene-1 (AEG-1) is a marker for aggressive salivary gland carcinoma. J Transl Med 9: 205, 2011. 
11. Liu L, Wu J, Ying Z, et al: Astrocyte elevated gene-1 upregulates matrix metalloproteinase- 9 and induces human glioma invasion. Cancer Res 70: 3750-3759, 2010.

12. Yoo BK, Emdad L, Su ZZ, et al: Astrocyte elevated gene-1 regulates hepatocellular carcinoma development and progression. J Clin Invest 119: 465-477, 2009.

13. Yu C, Chen K, Zheng H, et al: Overexpression of astrocyte elevated gene-1 (AEG-1) is associated with esophageal squamous cell carcinoma (ESCC) progression and pathogenesis. Carcinogenesis 30: 894-901, 2009.

14. Li J, Zhang N, Song LB, et al: Astrocyte elevated gene-1 is a novel prognostic marker for breast cancer progression and overall patient survival. Clin Cancer Res 14: 3319-3326, 2008.

15. Kikuno N, Shiina H, Urakami S, et al: Knockdown of astrocyteelevated gene-1 inhibits prostate cancer progression through upregulation of FOXO3a activity. Oncogene 26: 7647-7655, 2007.

16. Xu J-B, Wu H, He Y-L, Zhang C-H, Zhang, L-J, Cai S-R and Zhan W-H: Astrocyte-elevated gene-1 overexpression is associated with poor prognosis in gastric cancer. Med Oncol 28: 455-462, 2011

17. Chen W, Ke Z, Shi H, Yang S and Wang L: Overexpression of AEG-1 in renal cell carcinoma and its correlation with tumor nuclear grade and progression. Neoplasma 57: 522-529, 2010.

18. Song LB, Li W, Zhang HZ, et al: Over-expression of AEG-1 significantly associates with tumour aggressiveness and poor prognosis in human non-small cell lung cancer. J Pathol 219: 317-326, 2009.

19. Ash SC, Yang DQ and Britt DE: LYRIC/AEG-1 overexpression modulates BCCIPa protein levels in prostate tumor cells. Biochem Biophys Res Commun 371: 333-338, 2008.

20. Thirkettle HJ, Mills IG, Whitaker HC and Neal DE: Nuclear LYRIC/AEG-1 interacts with PLZF and relieves PLZF-mediated repression. Oncogene 28: 3663-3670, 2009.

21. Li J, Yang L, Song L, et al: Astrocyte elevated gene-1 is a proliferation promoter in breast cancer via suppressing transcriptional factor FOXO1. Oncogene 28: 3188-3196, 2009.

22. Santarpia M, Magri I, Sanchez-Ronco M, et al: mRNA expression levels and genetic status of genes involved in the EGFR and NF- $\mathrm{KB}$ pathways in metastatic non-small-cell lung cancer patients. J Transl Med 9: 163, 2011.

23. Li C, Li R, Song H, et al: Significance of AEG-1 expression in correlation with VEGF, microvessel density and clinicopathological characteristics in triple-negative breast cancer. J Surg Oncol 103: 184-192, 2011.
24. Hu GH, Chong RA, Yang QF, et al: MTDH activation by $8 \mathrm{q} 22$ genomic gain promotes chemoresistance and metastasis of poor-prognosis breast cancer. Cancer Cell 15: 9-20, 2009.

25. Liu H, Song X, Liu C, Li X, Wei L and Sun R: Knockdown of astrocyte elevated gene-1 inhibits proliferation and enhancing chemo-sensitivity to cisplatin or doxorubicin in neuroblastoma cells. J Exp Clin Cancer Res 28: 19, 2009.

26. Yoo BK, Gredler R, Vozhilla N, et al: Identification of genes conferring resistance to 5-fluorouracil. Proc Natl Acad Sci USA 106: 12938-12943, 2009.

27. Lee SG, Su ZZ, Emdad L, Sarkar D and Fisher PB: Astrocyte elevated gene-1 (AEG-1) is a target gene of oncogenic Ha-ras requiring phosphatidylinositol 3-kinase and c-Myc. Proc Natl Acad Sci USA 103: 17390-17395, 2006.

28. Lee SG, Su ZZ, Emdad L, Sarkar D, Franke TF and Fisher PB: Astrocyte elevated gene-1 activates cell survival pathways through PI3K-Akt signaling. Oncogene 27: 1114-1121, 2008.

29. Sarkar D, Park ES, Emdad L, Lee SG, Su ZZ and Fisher PB: Molecular basis of nuclear factor-kappaB activation by astrocyte elevated gene-1. Cancer Res 68: 1478-1484, 2008.

30. Emdad L, Sarkar D, Su ZZ, et al: Activation of the nuclear factor-kappaB pathway by astrocyte elevated gene-1: implications for tumor progression and metastasis. Cancer Res 66: 1509-1516, 2006

31. Xi W, Rui W, Fang L, Ke D, Ping G and Zhang H-Z: Expression of stathmin/op18 as a significant prognostic factor for cervical carcinoma patients. J Cancer Res Clin Oncol 135: 837-846, 2009.

32. Li B, Zhang LJ, Zhang ZL, et al: Synergistic tumor growth inhibition effect of prostate-specific antigen-activated fusion peptide BSD352 for prostate cancer therapy. Anticancer Drugs 22: 213-222, 2011.

33. Long M, Yin G, Liu L, et al: Adenovirus-mediated Aurora A shRNA driven by stathmin promoter suppressed tumor growth and enhanced paclitaxel chemotherapy sensitivity in human breast carcinoma cells. Cancer Gene Ther 19: 271-281, 2012.

34. Moore MA, Attasara P, Khuhaprema T, et al: Cancer epidemiology in mainland South-East Asia - past, present and future. Asian Pac J Cancer Prev 11 (Suppl 2): 67-80, 2010.

35. Folkman J: Tumor angiogenesis. Adv Cancer Res 43: 175-203, 1985. 